\title{
Primary mucinous adenocarcinoma of the eyelid
}

Abdulkader M. Albasri, MBBCh, PhD, Irfan A. Ansari, MBBS, MD, Abdulaziz R. Aljohani, MSc, Ahmed S. Alhujaily, MBBCh, KSUFP.

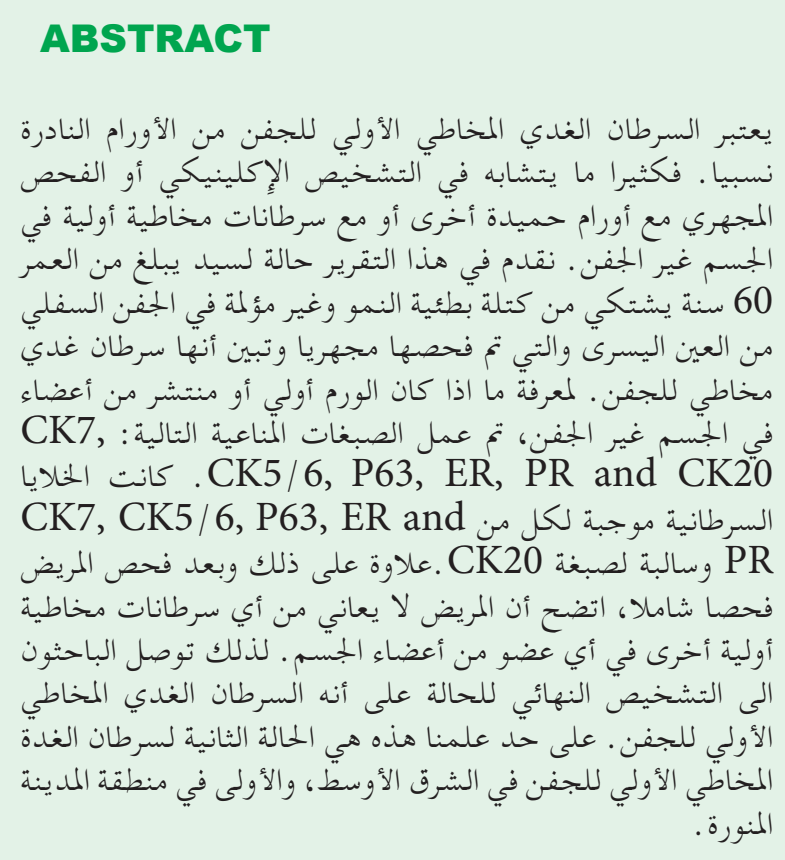

Primary mucinous adenocarcinoma (PMA) of the eyelid is an exceptionally rare clinical entity. Often, it mimics with benign lesions on clinical examination and with metastatic mucinous adenocarcinoma on histological examinations. We report a case of PMA in a 60-year-old male patient who came with a slowgrowing, painless swelling near the lower lid of the left eye. Excisional biopsy from the mass revealed a mucinous adenocarcinoma. To differentiate it from a metastatic mucinous adenocarcinoma, a wide range of immunohistochemistry panel was run. The tumor cells showed strong positivity for cytokeratin7, cytokeratin $5 / 6, p 63$, estrogen receptor, progesterone receptor and negativity for cytokeratin20. Moreover, extensive metastatic work-up did not show any primary malignancy elsewhere, hence a final diagnosis of PMA was made. We report a rare case of PMA from the Madinah region of Saudi Arabia.

Saudi Med J 2018; Vol. 39 (9): 940-945

doi:10.15537/smj.2018.9.22512

From the Department of Pathology (Albasri, Ansari), Taibah University, and from Department of Pathology (Aljohani, Alhujaily), King Fahad Hospital, Al-Madinah Al-Munawwarah, Kingdom of
Saudi Arabia.

Received 15th March 2018. Accepted 20th June 2018.

Address correspondence and reprint request to: Dr. Irfan A. Ansari, Department of Pathology, Taibah University, Al-Madinah AlMunawwarah, Kingdom of Saudi Arabia. E-mail: drirfanansari@ gmail.com

ORCID ID: orcid.org/0000-0002-5941-9768

$\mathrm{P}$ rimary mucinous adenocarcinoma (PMA) of the skin is an exceptionally rare clinical entity with an incidence of less than 0.1 cases per million individuals. Primary mucinous adenocarcinoma of the eyelid is even more rare both clinically and in the literature. ${ }^{1}$ It was first identified in 1952 by Lennox et al. ${ }^{2}$ Since then, less than 55 cases of PMA of the eyelid have been reported in the literature in English. ${ }^{3}$ Previously, this tumor had been reported under several names, including colloid, adenocystic, gelatinous and mucinous eccrine carcinoma. Historically, this tumor was classified as eccrine, but the World Health Organization (WHO) has classified PMA under both eccrine and apocrine differentiation. ${ }^{4}$ Clinically, PMA is characterized by an indolent course of a slow growing tan, gray to reddish in color, painless papillomatous, nodular or pedunculated lesion, and is often misdiagnosed as a simple cyst, chalazion, nevus or keratoacanthoma. At times, the tumor presents with ulceration, encrustation and brownish discoloration, which leads to an overt clinical diagnosis of melanoma, basal cell carcinoma or squamous cell carcinoma. Because of its variegated appearance on clinical examination, PMA of the eyelid is almost always diagnosed by complete histopathological examinations. ${ }^{5}$ Upon histological examination alone, differentiation between PMA and metastatic mucinous carcinoma, particularly from the breast and the gastrointestinal tract is near impossible. Hence, to establish an accurate diagnosis of PMA, immunohistochemical staining and a full body metastatic work-up are a must., Immunohistochemistry panels for this tumor have evolved over time with the final goal being having the 
minimum panel of stains. It has been reported that the cytokeratin panel is the most important marker for differentiating between primary and metastatic mucinous adenocarcinoma. ${ }^{7}$ The main objective of this case report is to highlight the rarity of the lesion and the importance of immunohistochemistry in differentiating PMA from metastatic mucinous adenocarcinoma.

Case Report. Patient information. A 60-yearold, otherwise healthy male patient presented with a painless swelling near the lower lid of his left eye at the ophthalmology clinic of Ohud hospital in Al-Madinah Al-Munawwarah, Kingdom of Saudi Arabia. At that time, he had had the swelling for almost 3 years. He had no similar swelling elsewhere in the body and no history of any malignancy in the past (Figure 1).

Clinical findings. On examination, the swelling was a superficial, non-tender, pink-colored nodule located horizontally on the tarsal plate of the left lower eyelid. It measured $2 \mathrm{~cm} \times 1.5 \mathrm{~cm} \times 1 \mathrm{~cm}$ and was mobile and firm in consistency. The swelling clinically resembled a benign lesion. The rest of the ocular examination was unremarkable in both eyes.

Diagnostic assessment. The excision biopsy of the lesion revealed a $2.5 \mathrm{~cm} \times 1.5 \mathrm{~cm} \times 1.3 \mathrm{~cm}$ mass. On the cut surface, a well-circumscribed, $2 \mathrm{~cm} \mathrm{x} 1 \mathrm{~cm} \times 0.8 \mathrm{~cm}$ grayish-white, firm tumor was seen in the center with 5 millimeter $(\mathrm{mm})$ surgical margins on all sides. A histological examination of the tumor showed overlying thinned-out epidermis, beneath which were seen sheets, cords and nodules of tumor cells lying in the pool of extracellular mucin (Figure 2A). At places, the cells showed cords, papillae and tubule formation, the tumor cells showed mild atypia and occasional mitotic figures; no necrosis was seen (Figure 2B), and all the surgical margins were free from the tumor. A diagnosis of mucinous adenocarcinoma was made. A wide range of immunohistochemistry panels were run to confirm the diagnosis. The tumor cells were strongly positive for cytokeratin 7 (CK7), cytokeratin 5/6 (CK5/6), tumor protein 63 (p63), estrogen receptor (ER), progesterone receptor (PR) and were negative for cytokeratin 20 (CK20) (Figure 3). To investigate the possibility that this tumor did not represent a metastatic mucinous

Disclosure. Authors have no conflict of interests, and the work was not supported or funded by any drug company. adenocarcinoma, the patient underwent a complete metastatic work-up which included, computed tomography (CT) of chest and abdomen, Positron emission tomography (PET) scan, esophago-gastroduodenoscopy and sigmoidoscopy; no abnormalities were detected. The final diagnosis of a Primary mucinous adenocarcinoma of the eyelid was given.

Therapeutic intervention. Considering the age of the patient and the long duration and gradually increasing size of the swelling, the patient was scheduled for a wide local excision with free surgical margins and referred to the King Fahad Hospital in Al-Madinah Al-Munawwarah.

Follow-up and outcomes. For the past year, the patient has been on a regular follow-up schedule and is clinically free from the disease at present.

Discussion. Primary mucinous adenocarcinoma can arise throughout the body, the most common sites being the head and the neck. The rate of occurrence of this tumor in the eyelid is reported by various previous studies to be between $30-45 \% .{ }^{1}$ Historically, PMA of the eyelid is a disease of Caucasians, as the prevalence is higher in the white population than in Asians or in African-Americans. ${ }^{8}$ An extensive search of the literature yielded only one article describing a case study of the first reported case of PMA of the eyelid from the Middle East, by Almater et al. ${ }^{6}$ Thus indicating the rarity of the lesion.

Primarily, PMA is a disease of elderly males, mostly seen in the fifth-seventh decade of life, with a mean patient age of 61 years. The age of the patient in our case was 60 years, which is comparable with the previous studies. Primary mucinous adenocarcinoma of the eyelid is often located on the lower lid. Contrary to the vertical growth pattern of sebaceous tumors, PMA frequently shows a horizontal growth pattern on the tarsal plate. ${ }^{5,6}$ The location in our case was also horizontal on the tarsal plate of the left lower lid. The origin of this tumor has long been discussed, and while some debate exists as to whether to classify it as having an eccrine or an apocrine origin, most authors have favored an eccrine origin based on immunohistochemical and ultrastructural features. ${ }^{6,7}$ Yet, some authors have reported favoring apocrine differentiation, however, the WHO has classified this tumor under both apocrine and eccrine differentiation. ${ }^{2}$

Clinically, these tumors usually present as slowgrowing, well-circumscribed, painless masses that have been present for several months to years, often ignored by the patients initially as they do not cause 


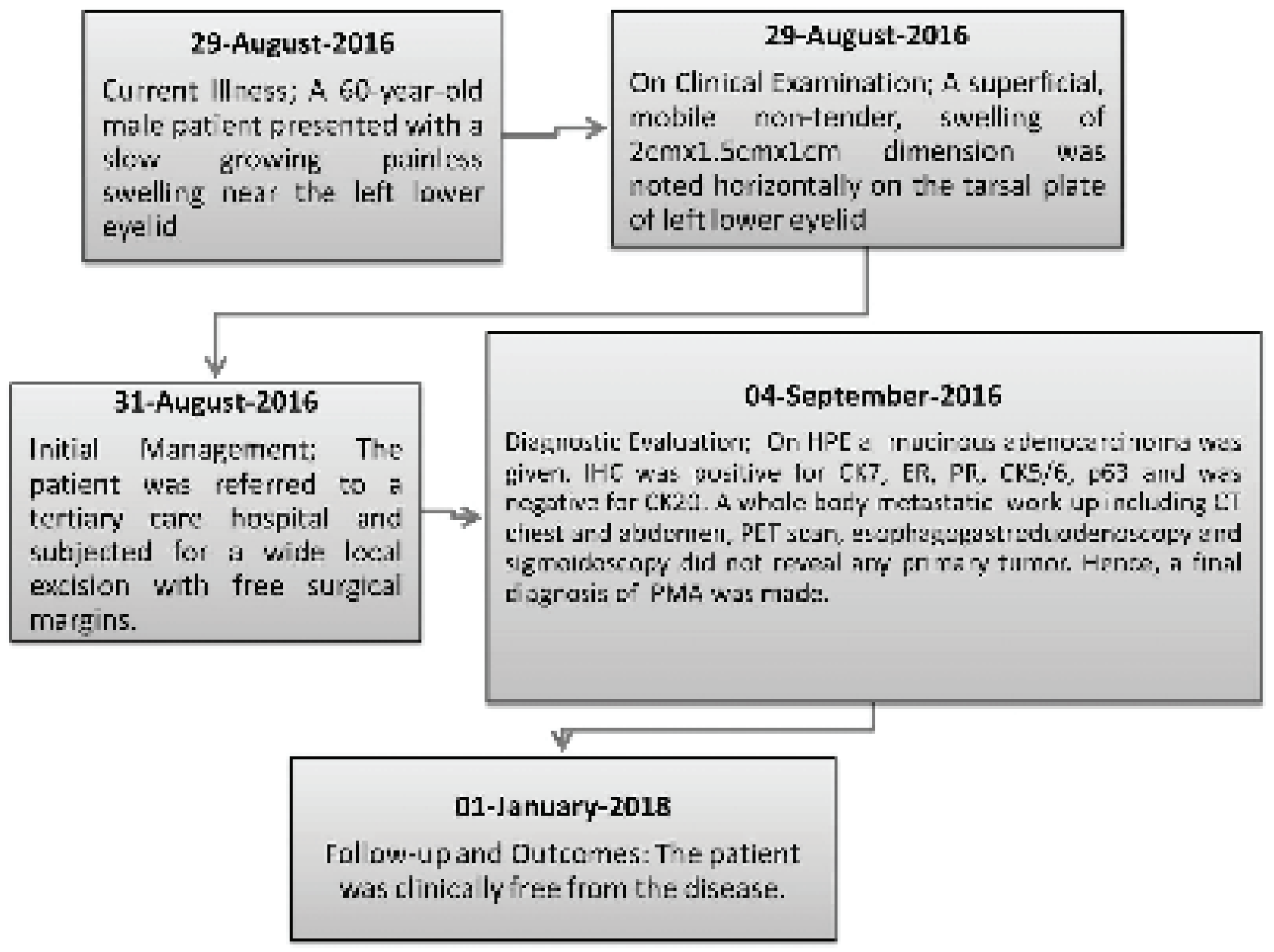

Figure 1 - Timeline summary of the patient's current illness, clinical examinations, diagnostic evaluations, therapeutic management and follow-up. HPE Histopathological examinations, IHC - Immunohistochemistry, CT - Computed tomography, PET - Positron emission tomography, PMA - Primary mucinous adenocarcinoma, CK7 - cytokeratin 7, CK5/6 - cytokeratin 5/6, p63 - tumor protein 63, ER - estrogen receptor, PR progesterone receptor.

any hindrance in the visual field. In our case, the tumor had been present for 3 years, and the patient came to the clinic mainly because of the gradual increase in the size of the tumor. On examination, the tumor is firm to cystic in consistency, often a papulonodular or pedunculated mass, and a tan to pink color. The size of such a tumor usually ranges between $1-8 \mathrm{~cm}$ with a mean diameter of $1.8 \mathrm{~cm}^{7}$ In our case, the maximum diameter of the tumor was $2 \mathrm{~cm}$, which is comparable with the previously reported data. Because of the nonspecific clinical presentation, the differential diagnoses often include an epidermal cyst, chalazion, basal cell carcinoma, keratoacanthoma, lacrimal gland carcinoma, melanocytic lesion, hemangioma, and metastatic carcinoma. ${ }^{4}$ As this tumor cannot be recognized precisely on clinical examination, PMA of the eyelid is almost always diagnosed on histopathological examinations. Our case also follows the benign look and average size on clinical examinations.

A careful histopathological examination is mandatory for a definitive diagnosis of PMA. On gross examination, it shows a firm-to-hard, grayish-white unencapsulated tumor mostly fixed to the dermis, thus making it difficult to be shelled out. The cut surface is usually gelatinous, although solid tumors with hemorrhage or purely cystic tumors have also been described. ${ }^{6}$ On histological examination, the classical features include the presence of dermal infiltration with an uninvolved overlying epidermis and adjacent adnexal structures, as seen in our case. The tumor cells 


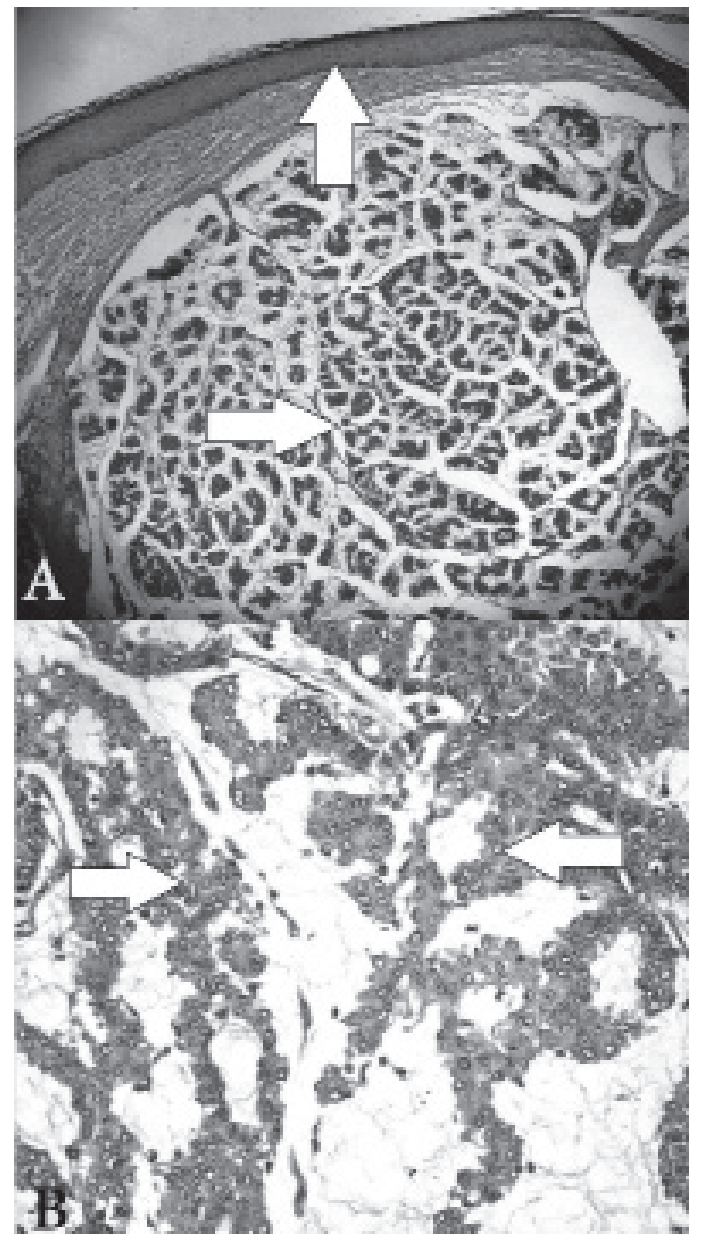

Figure 2 - Histopathology image of the tumor a) - Photomicrograph showing uninvolved epidermis (Vertical arrow), the dermis showing nests and clusters of tumor cells lying in the pool abundant mucin (Horizontal arrow ) (Hematoxylin \& Eosin 100X). b) The high power view of tumor showing cords, tubules and the papillae of tumor cells with minimal atypia and no necrosis lying in the pools of mucin (arrow), (Hematoxylin \& Eosin 400X).

are round to cuboidal with mild nuclear pleomorphism and occasional mitotic figures, and they are arranged in a glandular, micropapillary, or cribriform pattern lying characteristically in the lakes of mucin. Occasionally, the tumor cells are arranged in nests or duct-like structures, giving the appearance of eccrine or apocrine sweat glands. ${ }^{6,7}$ The histochemical reaction of this mucin is quite characteristic and might help in differentiating PMA from other closely resembling tumors like basal cell carcinoma, lacrimal gland tumors, and tumors producing metastatic mucin. The mucin of PMA is positive for periodic acid-Schiff (PAS), alcian blue (at $\mathrm{pH}$.4), mucicarmine, aldehyde fuchsin, and colloidal iron stains. The mucin is diastase and hyaluronidase resistant sialidase labile, thus making it nonsulfated sialomucin and distinguishable from hyaluronidasesensitive sulphomucin from other sweat gland tumors, basal cell carcinoma, lacrimal gland tumors, and metastatic mucinous adenocarcinoma of the gastrointestinal tract. ${ }^{7}$ However, special stains were not performed in our case. Other histopathological features differentiating PMA from metastatic adenocarcinoma from the gastrointestinal tract are the presence of highly pleomorphic cells, a dirty necrotic background, and goblet cell differentiation.

An accurate diagnosis of PMA needs other ancillary investigations, and the immunohistochemistry has greatly aided in identifying and differentiating PMA from metastatic mucinous adenocarcinoma. The expression pattern of a CK panel is very helpful. The PMA shows positive immunostaining with CK7 and a negative immunostaining for CK20, that is akin to the immunostaining pattern of the breast carcinoma, whereas metastatic adenocarcinoma from the gastrointestinal tract is CK7 negative and CK20 positive. Some other helpful immunohistochemical markers which point its origin from a secretory lobule include carcinoembryonic antigen, epithelial membrane antigen and CK AE1/E3. Gross cystic disease fluid protein-15 (GCDFP-15), estrogen receptor (ER) and progesterone receptor $(\mathrm{PR})$ are the other markers which also stain PMA and make it tougher to differentiate from a metastatic breast adenocarcinoma. Cytokeratin $5 / 6$ and p63 are the recently added markers which also help in differentiating PMA from metastatic mucinous adenocarcinoma, as proposed by various studies. ${ }^{1,6,7}$ Cytokeratin 5/6 and p63 primarily stain the myoepithelial cells and their expression in PMA confirms its adnexal origin. In the present case, the tumor cells were positive for CK7, ER, and PR and negative for CK20. In addition, the cells also showed the diffuse cytoplasmic and membranous positivity for CK5/6. It has been documented that CK5/6 is a relatively sensitive and specific marker in differentiating PMA from metastatic mucinous carcinoma. ${ }^{8}$ The role of p63 in the PMA is controversial; for instance, Pradhan et $\mathrm{al}^{9}$ have reported a negative p63 staining in their report, whereas several other authors have reported a positive staining for p63 in PMA. ${ }^{6-8}$ In our case, the p63 showed negative nuclear staining, which is contrary to the characteristic pattern of myoepithelial cells in PMA. Instead, p63 showed diffuse cytoplasmic positivity. This finding might indicate the aggressive behavior of the tumor as reported in other organs where cytoplasmic positivity of p63 is directly correlated with the 


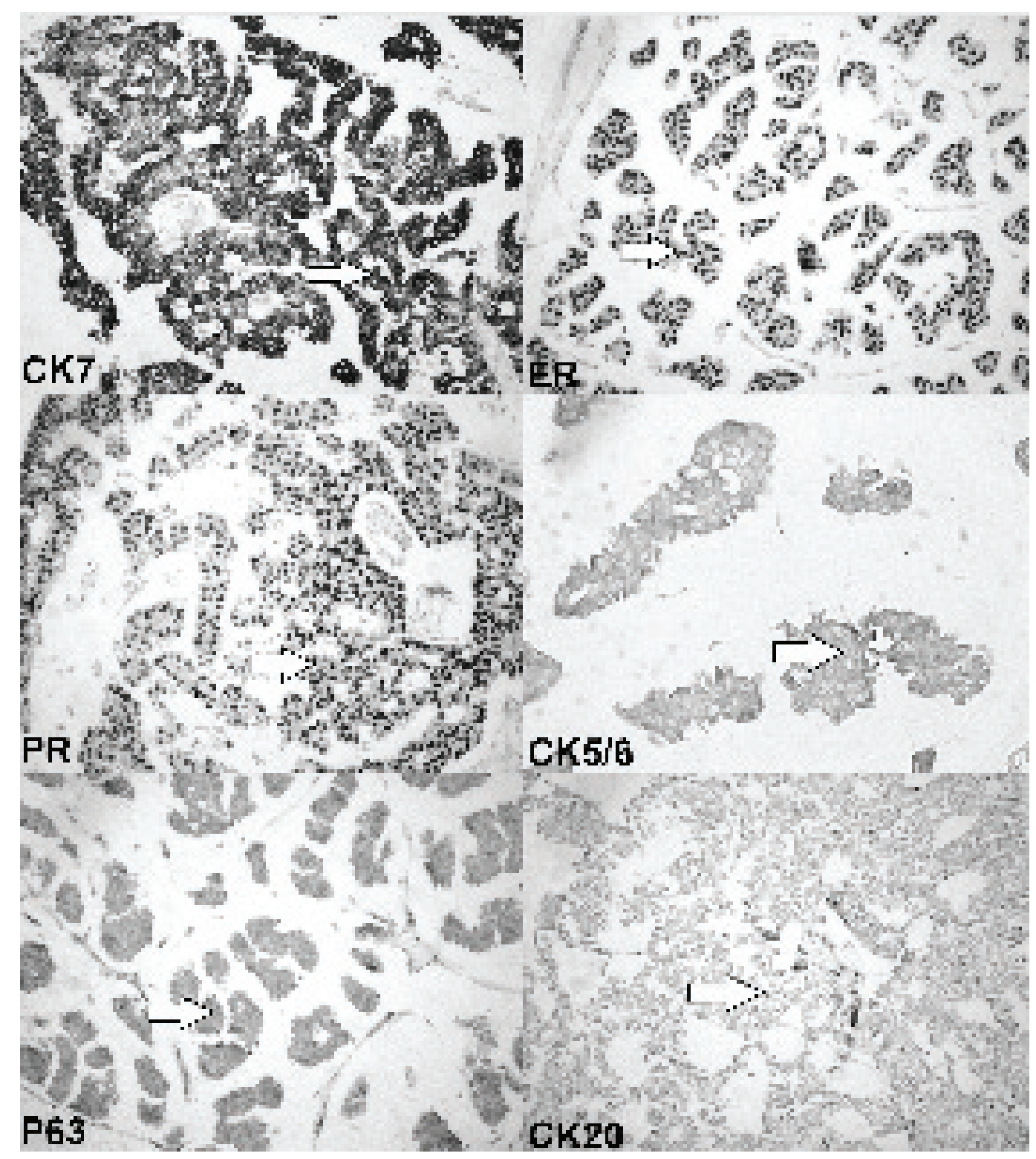

Figure 3 - Photomicrograph showing the sheets and clusters of tumor cells with strong immunoreactivity to CK7, CK7 - cytokeratin 7, CK5/6 - cytokeratin 5/6, P63 - tumor protein 63. ER - estrogen receptor, PR - progesterone receptor., cytoplasmic positivity to p63 and negative immunostaining for cytokeratin 20 - CK20 cytokeratin 20. (Immunohistochemistry stain 400X).

aggressive behavior and the worst prognosis. ${ }^{10}$ Our case, however, did not show any features of aggressiveness at the time of presentation and is disease free at one year of follow-up. Thus, the cytoplasmic staining in the PMA needs further exploration, as we did not find any evidence supporting our finding. Nevertheless, it is important to remember that these histological and immunohistochemical features are extremely helpful in the diagnosis of the primary etiology, but they surely cannot exclude the metastatic etiology. Hence, the full metastatic work-up remains the gold-standard approach to establish the diagnosis of PMA., ${ }^{6,7}$ Our patient also had a negative metastatic work-up for any primary malignancy elsewhere. Primary mucinous adenocarcinoma is a locally aggressive tumor with a high rate of recurrence. Hence, the mainstay of treatment for PMA is a wide local excision with free surgical margins. Because PMA's recurrence rate can reach up to $40 \%$, 
Mohs micrographic surgery could be an advantageous modality of treatment to avoid the recurrence. ${ }^{5}$ In our case, the patient was treated with a wide local excision with free surgical margins. The patient must be counseled for the regular follow-up to rule out any recurrence. For the past year, our patient has been on a regular follow-up schedule and is clinically free from the disease at present.

In conclusion, we report a rare case of PMA of the eyelid from the Westren region of Saudi Arabia. The PMA of the eyelid is a rare neoplasm, but before establishing this diagnosis, a primary mucinous carcinoma in the other organs must be ruled out. It is hard to make a definitive diagnosis of PMA merely on clinical and histological examinations. The wide range of immunohistochemical markers offers a promising tool for the differentiation between the primary and the metastatic mucinous adenocarcinoma. However, the role of p63 is still controversial and cytoplasmic staining of p63 is not yet reported in PMA. We strongly recommend further studies to explore the significance of cytoplasmic positivity of p63 in PMA.

Acknowledgment. The authors are thankful to Dr. Shabina Anjum, Assistant Professor, Department of Anatomy, Taibah University, Al-Madinah Al-Munawwarah, Kingdom of Saudi Arabia, for her continuous support and guidance in the drafting and critical evaluation of the manuscript. We would also like to extend our gratitude to Scribendi, the Editing and Proofreading Services for English Documents (https//www.scribendi.com) for the English language editing services.

\section{References}

1. Adefusika JA, Pimentel JD, Chavan RN, Brewer JD. Primary mucinous carcinoma of the skin: the Mayo Clinic experience over the past 2 decades. Dermatol Surg 2015; 41: 201-208.

2. Sanft DM, Zoroquiain P, Arthurs B, Burnier MN. Primary mucinous adenocarcinoma of the eyelid: A case-series. Human Pathology: Case Reports 2017; 9: 19-23.

3. Park JM, Kim SS. A Case of Primary Mucinous Adenocarcinoma of the Lower Eyelid. J Korean Ophthalmol Soc 2018; 59: 176-80.

4. Kamalpour L, Brindise RT, Nodzenski M, Bach DQ, Veledar E, Alam M. Primary cutaneous mucinous carcinoma: a systematic review and meta-analysis of outcomes after surgery. JAMA Dermatol 2014; 150: 380-384.

5. Almater AI, Alkatan HM, Alowais HA, Sheikh OA, Maktabi AM. Primary Mucinous Adenocarcinoma of the Eyelid: The First Reported Case in the Middle East. Adv Ophthalmol Vis Syst 2017; 7: 00247.

6. Beteddini OS, Sheikh S, Shareefi F, Shahab R. Primary mucinous adenocarcinoma of the scalp: A case report and literature review. Int J Surg Case Rep 2015; 10: 241-244.

7. Tak MS, Cho SE, Kang SG, Kim CH, Kim DW. Primary Cutaneous Mucinous Carcinoma of the Eyelid. Arch Craniofac Surg 2016; 17: 176-179.

8. Pradhan R, Pal S, Mondal PK, Mondal S. Primary mucinous eccrine carcinoma of axillary skin: report of a rare case. Indian J Dermatopathol Diagn Dermatol 2017; 4: 14-16.

9. Giannico GA, Ross HM, Lotan T, Epstein JI. Aberrant expression of $\mathrm{p} 63$ in adenocarcinoma of the prostate: a radical prostatectomy study. Am J Surg Pathol 2013; 37: 1401-1406.

\section{Copyright}

Whenever a manuscript contains material (tables, figures, etc.) which is protected by copyright (previously published), it is the obligation of the author to obtain written permission from the holder of the copyright (usually the publisher) to reproduce the material in Saudi Medical Journal. This also applies if the material is the authors own work. Please submit copies of the material from the source in which it was first published. 